\title{
New disease gene location and high genetic heterogeneity in idiopathic scoliosis
}

\author{
Patrick Edery $^{\star, 1,2}$, Patricia Margaritte-Jeannin ${ }^{3,4}$, Bernard Biot ${ }^{5}$, Audrey Labalme ${ }^{1}$, Jean-Claude Bernard ${ }^{5}$, \\ Joëlle Chastang ${ }^{1}$, Behrouz Kassai ${ }^{6}$, Marie-Helene Plais ${ }^{7}$, Florina Moldovan ${ }^{8}$, Francoise Clerget-Darpoux ${ }^{9,10}$
}

Idiopathic scoliosis (IS) is a spine disorder of unknown origin with 1.5-3\% prevalence in the general population. Besides the large multifactorial-form sample of IS, there is a good evidence for the existence of a monogenic subgroup in which the disease is inherited in a dominant manner. However, results from literature suggest a strong heterogeneity in the locations of the mutated genes. Using a high-resolution genome-wide scan, we performed linkage analyses in three large multigenerational IS families compatible with dominant inheritance including 9-12 affected members or obligate carriers. In two of these families, our results suggested intra-familial genetic heterogeneity, whereas, in the other, we observed a perfect marker disease co-segregation in two regions at 3q12.1 and 5q13.3. We can state that one of these two locations is a novel IS disease gene locus, as the probability of having this perfect co-segregation twice by chance in the genome is very low $(P=0.001)$. Lastly, in all three families studied, linkage to the previously mapped dominant IS loci on chromosomes $19 \mathrm{p} 13.3,17 \mathrm{p} 11.2$, $9 q 34,17 q 25$ and $18 q$ is unlikely, confirming that there is a high genetic heterogeneity within the subgroup of dominant forms of IS.

European Journal of Human Genetics (2011) 19, 865-869; doi:10.1038/ejhg.2011.31; published online 16 March 2011

Keywords: idiopathic scoliosis; complex disorder; high-density genome-wide linkage; genetic mapping; chromosomes 5q13-q14; 3q11.1-q13.2

\section{INTRODUCTION}

Idiopathic scoliosis (IS) is a spine deformity of unknown cause commonly defined by a rotation of vertebral bodies, leading to a structurally fixed lateral curvature of the spine with a Cobb's angle of at least $10^{\circ}$. IS affects up to $3 \%$ of the population, ${ }^{1}$ girls being more commonly affected than boys. ${ }^{2,3}$ Adolescent idiopathic scoliosis (AIS, MIM 181800) occurs in otherwise healthy adolescents and accounts for $\sim 85 \%$ of IS cases, other cases being infantile or juvenile (15\%). About 3 per 1000 children present with severe AIS forms over $30^{\circ}$, which may require braces or surgical treatment, and may have severe functional and aesthetic consequences.

Aside from idiopathic forms, scoliosis may also occur secondary to other hereditary disorders, including defects in extracellular matrix components (eg, Marfan syndrome and Ehlers-Danlos syndromes), neuromuscular disorders (eg, Friedreich ataxia and muscular dystrophies), osteochondrodystrophies (eg, osteogenesis imperfecta and neurofibromatosis), vertebral segmentation defects or other malformation syndromes including CHARGE syndrome and SmithMagenis (del17p11.2) syndrome.

Several population studies established that up to $40 \%$ of IS are familial $^{4}$ and that there is a high concordance rate of this disease in monozygotic twins. ${ }^{5}$ Many studies concluded that the most likely mode of inheritance was multifactorial, postulating that predisposing alleles were required together with environmental factors for phenotypic expression. ${ }^{6}$ Although the exact mode of inheritance remains unknown in many IS cases, there is now evidence for a monogenic subgroup. A segregation analysis using a model with age and gender effects established a significant contribution of a major causal gene transmitted according to an autosomal dominant inheritance with sex-dependent incomplete penetrances. ${ }^{7}$ A small number of model-dependent linkage analyses using an autosomal dominant model in large multigenerational IS families have been performed successfully to date. Indeed, such large multiplex IS families compatible with dominant inheritance are very rare. A genome-wide scan of seven unrelated multiplex families of Chinese descent with AIS concluded that there was a linkage to chromosome 19p13.3 (IS1, MIM 181800). ${ }^{8}$ This finding was replicated in several IS multiplex families in Alden et al's study. ${ }^{9}$ A similar study in an Italian family mapped a second locus to $17 \mathrm{p} 11.2$ (IS2, MIM 607354). ${ }^{10}$ Another study reported on the mapping of two loci for IS on chromosome 9q31.2-q34.2 (IS4, MIM 612238) and 17q25.3-qtel (IS5, MIM 612239), the latter by combining information obtained from two unrelated IS families. ${ }^{11}$ Recently, a locus for IS and pectus excavatum was found in a single large family on chromosome $18 \mathrm{q} .{ }^{12}$

In the present study, we performed model-dependent linkage analyses in three large IS families compatible with autosomal dominant inheritance of the disease trait with a high number of informative meioses. We compared our results with published linkage data.

\footnotetext{
${ }^{1}$ Hospices Civils de Lyon, Service de Cytogénétique Constitutionnelle, Bron, France; ${ }^{2}$ Université Lyon 1, Centre de Recherche en Neurosciences, Inserm U1028, CNRS UMR5292, Lyon, France; ${ }^{3}$ INSERM, UMR_S946, Paris, France; ${ }^{4}$ Université Paris Diderot, Paris, France; ${ }^{5}$ Centre de Réadaptation Fonctionnelle des Massues, Lyon, France; ${ }^{6}$ Hospices Civils de Lyon, Centre d'Investigation Clinique, Bron, France; ${ }^{7}$ Fondation Cotrel-Institut de France, Paris, France; ${ }^{8} \mathrm{CHU}$ Sainte Justine, Centre de Recherche, Faculte

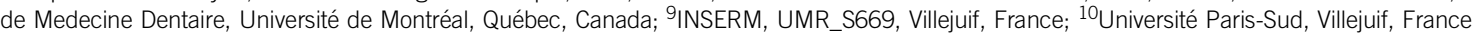

*Correspondence: Professor P Edery, Hospices Civils de Lyon, Service de Cytogénétique Constitutionnelle, Groupement Hospitalier East, CBPE 6eme etage, 59 Boulevard Pinel, Bron Cedex 69677, France. Tel: +334 7212 9698; Fax: +334 7212 9710; E-mail: patrick.edery@chu-lyon.fr or charles.edery@chu-lyon.fr
}

Received 15 September 2010; revised 15 January 2011; accepted 28 January 2011; published online 16 March 2011 


\section{FAMILIES AND METHODS}

\section{Patient and family inclusion}

We selected large multiplex IS families, and we genotyped the largest families, that is, those including the highest number of affected individuals or obligate carriers. A positive diagnosis for IS was reached by combining clinical examination of the spine, including the forward bending test (Adams test), and the Cobb's angle measurement on radiographic evaluation. Both Cobb's angle above $15^{\circ}$ (to minimise the risk of phenocopies) and vertebral rotation were required for the positive diagnosis of IS. Patients harbouring a spine curvature but no rotational component were assigned an 'unknown status', as were those with any other associated anomaly of the spine or, more generally, with atypical IS. Patients with normal clinical examination and strictly normal spine on radiograph were considered 'unaffected'. All individuals underwent clinical examination by at least one clinical geneticist (PE) and one orthopaedist (BB and/or JCB). Each radiograph was carefully and often repeatedly checked by the clinical geneticist and one or both orthopaedists.
Pedigrees of the three selected IS families are shown in the Figure 1. All families are three-generation pedigrees, suggestive of the transmission of an autosomal dominant mutation with incomplete penetrance. Clinical data on patients belonging to the families studied are provided in Table 1.

\section{DNA sample collection}

We selected 57 individuals for genotyping, including 27 affected patients or obligate carriers belonging to the three families presented here. Blood samples were collected from each participant (IS patients and their first-degree affected or unaffected relatives) after providing them with an information sheet describing the research study and obtaining their informed written consent. This research study was approved by the local ethical committee and was promoted by the Hospices Civils de Lyon, France. Genomic DNA was extracted from blood samples according to standard procedures. EBV transformation of lymphocytes was performed in at least one affected individual per family. After the cell lines were established, transformed lymphocytes

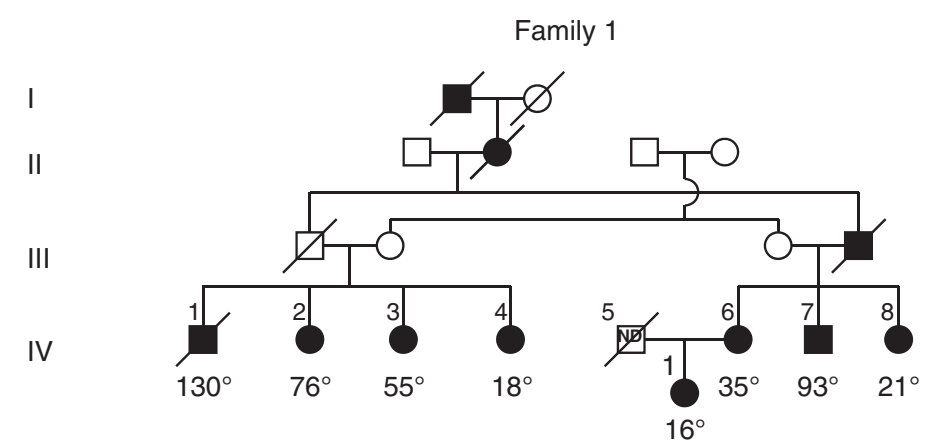

Family 2
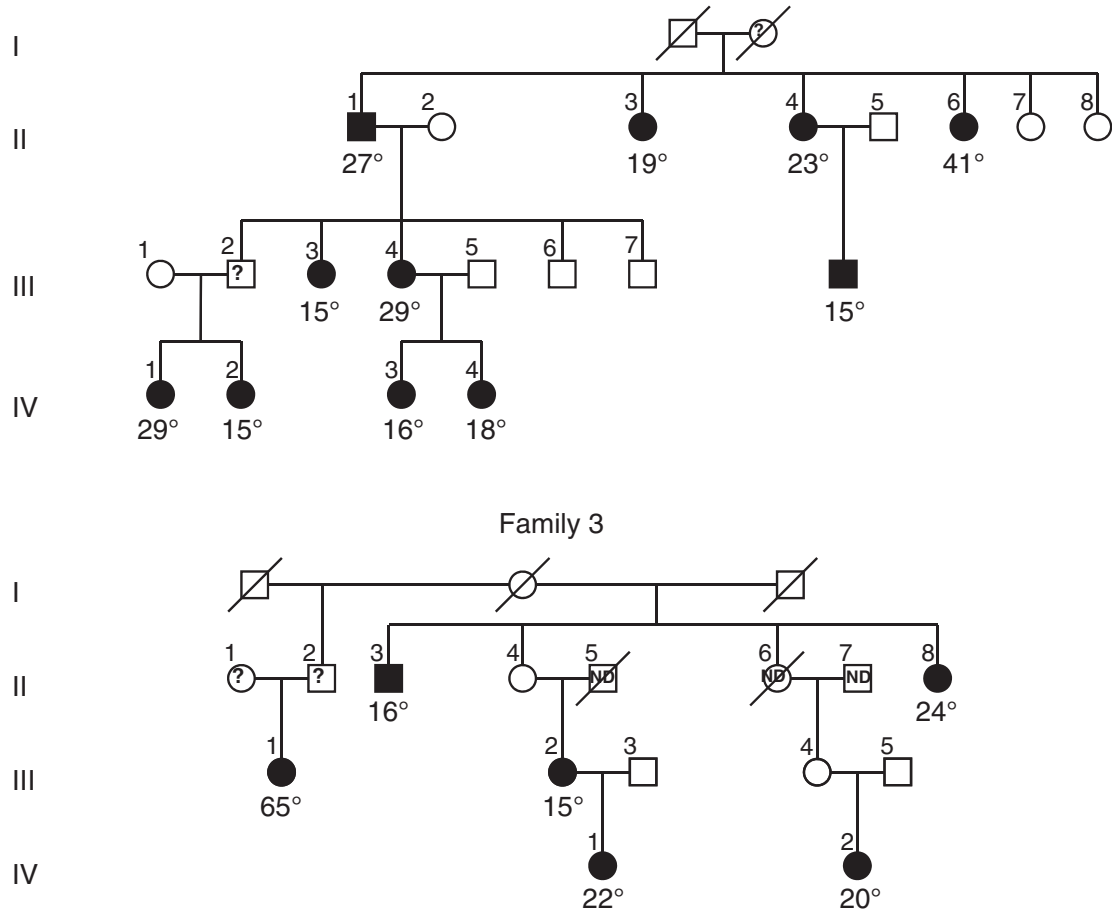

Figure 1 Simplified pedigrees are drawn for families 1-3. All pedigrees are compatible with autosomal dominant inheritance of the disease trait. Cobb's angles are indicated. Question marks indicate uncertain status. Several unaffected individuals who have been genotyped are not included, as they were not useful in linkage analysis. In family 2, four unaffected individuals are included, as they contributed to the mod score analysis. ND, status not determined. 
Table 1 Clinical data for patients included in this study

\begin{tabular}{|c|c|c|c|c|c|c|}
\hline Family & Patient & $\begin{array}{l}\text { Age at diagnosis } \\
\text { (years old) }\end{array}$ & $\begin{array}{c}\text { Cobb's angle on radiograph } \\
\text { (age: years old) }\end{array}$ & Cobb's angle & $\begin{array}{l}\text { Apical } \\
\text { vertebrae }\end{array}$ & Therapy \\
\hline \multirow[t]{7}{*}{$\mathrm{F} 1$} & IV-2 & 13 & $76^{\circ}(65)$ & Right thoracic & D9 & Physiotherapy \\
\hline & IV-3 & Adolescence & $55^{\circ}(61)^{a}$ & Right thoracolumbar & D12 & Spinal surgery \\
\hline & IV-4 & Fortuitous & $18^{\circ}(50)$ & Right lumbar & L2 & None \\
\hline & IV-6 & 12 & $35^{\circ} / 28^{\circ}(64)$ & Right thoracic/left lumbar b & D8-D9/L2-L3 & Bracing \\
\hline & IV-7 & 6 & $90^{\circ} / 93^{\circ}(20)$ & Right thoracic/left lumbarb & D7/L2 & Unknown \\
\hline & IV-8 & 9 & $21^{\circ}(26)^{a}$ & Right thoracic/left lumbar ${ }^{b}$ & D8/L3 & Spinal surgery \\
\hline & V-1 & 15 & $16^{\circ}(15)$ & Left lumbar & L3 & None \\
\hline \multirow[t]{11}{*}{$\mathrm{F} 2$} & II-1 & Fortuitous & $27^{\circ}(73)$ & Right thoracolumbar & D12-L1 & None \\
\hline & $11-3$ & Fortuitous & $19^{\circ}(74)$ & Right lumbar & L1 & None \\
\hline & $\| 1-4$ & Fortuitous & $23^{\circ}(71)$ & Right lumbar & L2 & None \\
\hline & $11-6$ & Adolescence & $41^{\circ}(67)$ & Right thoracolumbar & L1 & None \\
\hline & III-3 & Adolescence & $15^{\circ}(49)$ & Right thoracolumbar & L1 & Bracing \\
\hline & III-4 & Fortuitous & $29^{\circ}(46)$ & Right thoracolumbar & D12 & None \\
\hline & III-8 & Fortuitous & $15^{\circ}(42)$ & Right thoracolumbar & L1 & None \\
\hline & IV-1 & adolescence & $29^{\circ} / 29^{\circ}(20)$ & Right thoracic/left lumbarb & D8/L2 & Physiotherapy \\
\hline & IV-2 & fortuitous & $15^{\circ}(16)$ & Right thoracic & D7 & None \\
\hline & IV-3 & fortuitous & $14^{\circ} / 16^{\circ}(19)$ & Right thoracic/left lumbarb & D8/L1 & None \\
\hline & IV-4 & 12 & $18^{\circ}(15)$ & Left thoracolumbar & L1 & Physiotherapy \\
\hline \multirow[t]{6}{*}{ F3 } & $11-3$ & fortuitous & $14^{\circ} / 16^{\circ}(58)$ & Right thoracic/left lumbarb & D8/L1 & None \\
\hline & $11-8$ & fortuitous & $24^{\circ}(66)$ & Right thoracic & D8 & None \\
\hline & III-1 & adolescence & $65^{\circ}(18)$ & Right thoracic left lumbar & D8 & Spinal surgery \\
\hline & III-2 & fortuitous & $15^{\circ}(32)$ & Right lumbar & L2 & None \\
\hline & IV-1 & 9 & $22^{\circ}(15)$ & Right thoracic & D8 & Physiotherapy \\
\hline & IV-2 & 10 & $20^{\circ}(15)$ & Right thoracolumbar & L1 & Bracing \\
\hline
\end{tabular}

aCobb's angle after arthrodesis.

bouble major curve.

were stored frozen in liquid nitrogen. Normal standard chromosomal studies on blood lymphocytes were obtained from at least one affected individual in each family ruling out gross chromosomal abnormalities.

\section{Genotyping}

Considering the large number of possible IS candidate genes with regard to their function and the wide genetic heterogeneity of IS, we decided to favour genome-wide approaches rather than candidate loci-mapping strategies. We genotyped the three large multiplex IS families using a high-density 1000 microsatellite DNA marker set, with an average spacing of $3.7 \mathrm{~cm}$ between each marker, where genetic locations are based on the deCODE map. ${ }^{13}$

\section{Statistical analyses}

The classical method used for mapping a disease gene under monogenic transmission is the Morton Lod Score analysis (1955). A lod score value of 3.00 (for autosomal loci) means that the hypothesis of linkage between the disease trait locus and the marker locus is 1000 times more likely than the hypothesis of no linkage. This method is very powerful when the disease is due to a mutation of the same gene in all families with full penetrance for mutation carrier. In the present situation, it is realistic to assume that the disease is due to segregation of a dominant mutation in the families selected for this study. However, the results from literature suggest a strong heterogeneity in the mutated genes. In addition, penetrance values may differ according to the genes and mutations involved. Clerget-Darpoux et al ${ }^{14}$ showed that a misspecification of the penetrance values results in a lod score decrease and hence in a loss of the power to detect linkage.

To overcome the inter-familial genetic heterogeneity, we performed a lod score analysis separately in each family. Analyses were performed assuming the segregation of a dominant mutation, with and without the possibility of phenocopies. In linkage analysis, marker segregation was considered only in affected individuals. The status of other family members, including unaffected individuals, was assumed to be unknown. In such an analysis, the results do not depend on the absolute penetrance values, but only on the relative ones (probability to be affected for a non-mutation carrier versus this probability for a mutation carrier).

In the first step, we measured in each family the lod score value that could be reached at the disease gene locus in the situation of no phenocopy and full informativity. This theoretical maximum lod score value $Z_{\text {imax }}$ can be simply inferred from the number Ti of disease allele transmissions to affected individuals in each family. If we denote Mi the number of meioses between a common ancestor and all affected, ni the number of common ancestors and $\mathrm{Ti}=\mathrm{Mi}-\mathrm{ni}$, in each family, Fi:

$$
Z_{\text {imax }}=\log _{10}(L(\theta=0) / L(\theta=0.5))^{\mathrm{Ti}}=-\mathrm{Ti} \times \log _{10}(1 / 2)
$$

where $L(\theta=0)$ is the likelihood of $\mathrm{H} 1$ (hypothesis of linkage at $\theta=0$, that is, no recombination event between the marker and the disease locus) and $L(\theta=0.5)$ is the likelihood of $\mathrm{H} 0$ (hypothesis of no linkage).

In the second step, we estimated the probabilities under $\mathrm{H} 0$ to reach the maximum lod score on each arm of autosomes 0,1 or more than once. This maximum occurs according to a Poisson distribution with a parameter $\lambda$ equal to the probability of reaching $Z_{\text {imax }}$ if hypothesis $\mathrm{HO}$ is true $=(0.5)^{\mathrm{Ti}}$. On the whole genome (44 chromosome arms), this parameter $\lambda$ is equal to $44 \times(0.5)^{\mathrm{Ti}}$.

In a third step, we computed maximum Morton lod scores using Merlin software ${ }^{15}$ separately in each of the selected families. 
In the final step, we tested in each of the three families studied the compatibility with linkage to the five loci previously considered to be IS loci (19p13.3, 17p11.2, 9q34, 17q25 and 18q) using both the genetic model without phenocopy described here and a more flexible model allowing for various rates of phenocopies $(0.01,0.03,0.10$ and 0.20$)$.

\section{RESULTS}

Table 2 gives the values Mi, ni, Ti and the expected maximum lod score $Z_{\text {imax }}$ in each family Fi. It also gives the probabilities to reach it 0 , 1 or more than once under the $\mathrm{H} 0$ hypothesis in whole-genome analysis. Expected maximum lod scores are 1.51, 3.01 and 2.71 in families F1, F2 and F3, respectively. The probability of never reaching $Z_{\text {imax }}$ under $\mathrm{H} 0$, and thus only once (under $\mathrm{H} 1$ ), in whole-genome analysis is $25 \%$ for F1, $96 \%$ for $\mathrm{F} 2$, and $92 \%$ for $\mathrm{F} 3$.

Table 3 gives, for each family F1, F2 and F3, the maximum lod score $(\max Z)$ and marker informativity at this location. In families F1 and $\mathrm{F} 3$, the maximum lod scores obtained after whole-genome scan were significantly lower than the expected $Z_{\max }$ previously estimated. Affected individuals never shared a same transmitted allele in any chromosome arm. Consequently, the hypothesis that the disease is due to the transmission of a dominant mutation has to be reconsidered in these families. The variable severity in these two families suggests a possible intra-familial genetic heterogeneity. In family F1, the severity of IS in affected individuals is highly variable, as, in several patients of this family, Cobb's angles are between $16^{\circ}$ and $24^{\circ}$, and over $50^{\circ}$ in four patients. Similarly, patient III1 of family F3 is affected with a severe form of IS (Cobb's angle of $65^{\circ}$ ), whereas angles for the five other patients are between $15^{\circ}$ and $24^{\circ}$. When patient III1 of family F3 is removed from the linkage study, the lod score reaches the theoretical maximum value of 2.11 at the D15S979 locus.

In family $\mathrm{F} 2$, the lod score $Z_{\text {imax }}$ of 3.01 is reached both on chromosomes 3 (3q12.1) and 5 (5q13.3). As noted in Table 2, the probability of obtaining this value twice randomly (under $\mathrm{H} 0$ ) is very small ( $P=0.001)$. It means that there is strong evidence that a disease gene lays in one of these two regions. The lengths of chromosome intervals shared by all patients at the two possible disease gene locations are 7 and $12 \mathrm{cM}$, respectively (Table 3 ). The boundaries of these intervals, (D3S3690-D3S3045) and (D5S2851-D5S1397), respectively, were deduced from recombination events in affected individuals as observed on haplotypes. To test whether the study of unaffected members of family F2 may help in the discrimination between these two locations, we performed a mod score analysis ${ }^{16}$ with all genotyped family members (affected and non-affected) using the software Genehunter-Modscore 2.0.1. ${ }^{17}$ The results from four unaffected individuals in this family, II7, II8, III6 and III7, (see the Figure 1) proved to be informative. The $\bmod$ scores obtained at chromosome 3 and 5 loci, respectively, were identical and did not allow us to discriminate between the two possible locations.

Lastly, we considered lod scores obtained in families F1, F2 and F3 on the five regions claimed to be IS loci. All lod scores were negative when assuming no phenocopy, and a maximum value of 0.00 was reached when the model allowed for phenocopies.

\section{DISCUSSION}

Using a set of 1000 polymorphic markers (decode set), we performed a genome-wide linkage analysis under a dominant mode of transmission in three large multiplex IS families. Several pitfalls, such as interfamilial genetic heterogeneity, possible phenocopies or unknown penetrance for mutation carrier, were taken into account. First, lod score analyses were computed for each family independently. Second, to decrease the risk of phenocopies, individuals with a Cobb's angle of at least $15^{\circ}$, instead of the widely accepted threshold of $10^{\circ}$, were considered as affected. Indeed, mild scoliosis or even mild curvature and/or rotation of the spine are likely to be caused by a combination of environmental and minor gene effects rather than by a major gene effect. ${ }^{7}$ In addition, previous reports and our own observations showed that there are important variations in Cobb's angle measurements of up to $5^{\circ}$ among different physicians. ${ }^{18,19}$

This study shows the mapping of a new locus for dominant IS in a large family (F2) either on $5 \mathrm{q} 13-\mathrm{q} 14$ or on $3 \mathrm{q} 11-\mathrm{q} 13$. Indeed, in this family, the observed $Z_{\max }$ reached the expected $Z_{\max }$ value of 3.01 on both genomic locations. As the probability of obtaining a $Z_{\max }$ value in the genome twice by chance is very low $(P=0.001)$, we can conclude that there is strong evidence that a novel IS disease gene lies in one of these two locations. This IS gene still remains to be identified. Among the genes in the two chromosomal regions, many are involved in

Table 2 Informativity studies performed in each family

\begin{tabular}{|c|c|c|c|c|c|c|c|}
\hline \multirow{2}{*}{$\begin{array}{l}\text { Family } \\
(\mathrm{Fi})\end{array}$} & \multirow{2}{*}{$\begin{array}{c}\text { No. of meioses } \\
\text { between a common ancestor } \\
\text { and all affected individuals } \\
(\mathrm{Mi})\end{array}$} & \multirow{2}{*}{$\begin{array}{c}\text { Number of } \\
\text { common ancestors } \\
\text { (ni) }\end{array}$} & \multirow{2}{*}{$\begin{array}{l}\text { Number of disease } \\
\text { allele transmissions to } \\
\text { affected individuals } \\
\quad(T i=M i-n i)\end{array}$} & \multirow{2}{*}{$\begin{array}{c}\text { Expected maximum } \\
\text { lod score } \\
\left(\mathrm{Z}_{\text {imax }}=-T_{i} \log _{10}(0.5)\right)\end{array}$} & \multicolumn{3}{|c|}{$\begin{array}{c}\text { Probabilities to reach } \mathrm{Z}_{\text {imax }} \\
\text { under } \mathrm{HO}, 0,1 \text { or more than once } \\
\text { in whole-genome analysis }\end{array}$} \\
\hline & & & & & 0 & 1 & $>1$ \\
\hline $\mathrm{F} 1$ & 9 & 4 & 5 & 1.51 & 0.253 & 0.346 & 0.401 \\
\hline $\mathrm{F} 2$ & 12 & 2 & 10 & 3.01 & 0.958 & 0.041 & 0.001 \\
\hline F3 & 10 & 1 & 9 & 2.71 & 0.918 & 0.079 & 0.003 \\
\hline
\end{tabular}

Only affected individuals have been taken into account.

Table 3 Maximum lod score values obtained in each family using only affected individuals

\begin{tabular}{lcccc}
\hline Family & Expected $Z_{\max }$ & Observed $Z_{\max }$ & Informativity & Localisation (interval length) \\
\hline F1 & 1.51 & 0.85 & 0.79 & D14S274 \\
F2 & 3.01 & 3.00 & 0.99 & D5S2003 (D5S2851-D5S1397, 12cM) \\
& 3.01 & 3.01 & 0.99 & D3S2462 (D3S3690-D3S3045, 7cM) \\
F3 & 2.71 & 1.23 & 0.87 & D15S979 \\
\hline
\end{tabular}


cellular signalling, some are coding for hypothetical/uncharacterised proteins yet to be discovered and others were cloned but their function is not yet fully documented. Mutation screening using either exome sequencing or targeted high-throughput sequencing, gene expression studies and in vivo functional analysis of candidate genes within these loci need to be performed.

In this study, we also provide further evidence for a high heterogeneity among monogenic forms of IS. In each of the three families studied, the lod scores are either negative or inconclusive, depending on the phenocopy rate assumption, at the five previously suggested locations for IS genes, namely, 19p13.3, 17p11.2, 9q34, 17q25 and 18q. Such high genetic heterogeneity favours the view that computing IS families independently, when performing linkage analyses based on a monogenic model hypothesis, is critical to obtaining accurate results.

We also show that the selection of multiplex IS families diagnosed with strict clinical criteria does not warranty that the apparently dominant inheritance is simply explained by the segregation of a unique gene mutation. We have to consider the difficulty of selecting families with a high number of informative meioses, without introducing intra-familial heterogeneity or phenocopies, to be able to detect linkage at a family level. It seems that the criterion of a minimum angle of $15^{\circ}$ is not sufficient to ensure intra-familial genetic homogeneity, and we hypothesise that clinical variability between family members also has to be minimised. Indeed, in both families $\mathrm{F} 1$ and $\mathrm{F} 3$, the $Z_{\max }$ values obtained were far beyond the values expected under the assumption of a single causative mutation segregating in all affected members. In family F1, IS patients have a wide range of Cobb's angle values extending from $16^{\circ}$ to $130^{\circ}$, and in family $\mathrm{F} 3$, one patient is affected with a severe form of IS (Cobb's angle of $65^{\circ}$ ), whereas angles for the five other patients are between $15^{\circ}$ and $24^{\circ}$. When the patient with the Cobb's angle of $65^{\circ}$ from family F3 is removed from the analysis, we observe a perfect co-segregation of the disease and the D15S979 marker. Therefore, in such studies, we suggest paying careful attention to the clinical aspects of scoliosis, possibly including Cobb's angle values, even within single families. Such stratification by the severity of the phenotype or by other discrete clinical aspects of scoliosis may minimise the risk of intra-familial heterogeneity. The results provided in our study, supporting a high degree of inter- and intra-familial genetic heterogeneity in IS, raise questions regarding several previously reported mapping studies based on linkage data obtained by pooling together independent IS families. It should be noted that a posteriori selection of families compatible with linkage to a specific locus may lead to false primary linkage and/or fine mapping. Thus, in genetically heterogeneous disorders in which a different mutated gene in each family may be dealt with, pooling linkage data obtained from unrelated families should be considered with caution. Accurate linkage-mapping studies are required to allow the subsequent identification of dominant IS genes. To date, no dominant IS gene has been identified, and we hope that the present study will prove to be an important step towards this goal. Also, although dominant IS genes, yet to be identified, may account for a restricted number of IS cases, they may provide insights into the genetic bases of multifactorial IS, which remain largely unknown. ${ }^{20,21}$ Future genetic and functional analyses of candidate genes located within these defined regions will hopefully reveal gene defects accounting for IS, which will allow, in turn, the identification of secondary molecular factors that may point towards yet unrecognised IS pathophysiological pathways.

\section{CONFLICT OF INTEREST}

The authors declare no conflict of interest.

\section{ACKNOWLEDGEMENTS}

We thank the patients who participated to this study. We also thank Heather Yampolsky for her editorial assistance. This work was supported by the Fondation Yves Cotrel-Institut de France, the Hospices Civils de Lyon (PHRC regional 2001), the Fondation pour la Recherche Médicale, the Rhône-Alpes region (contract Emergence), the University Lyon 1 and the Association GEN'HOM.

\section{WEB RESOURCES}

Online Mendelian (OMIM), http://www.ncbi.nlm.nih.gov/sites/entrez?db=omim National Center for Biotechnology Information, http://www.ncbi.nlm.nih.gov/ deCODE Genetics, http://www.decode.com/

1 Rogala EJ, Drummond DS, Gurr J: Scoliosis: incidence and natural history. A prospective epidemiological study. J Bone Joint Surg Am 1978; 60: 173-176.

2 Shands Jr AR, Eisberg HB: The incidence of scoliosis in the state of Delaware; a study of 50000 minifilms of the chest made during a survey for tuberculosis. J Bone Joint Surg Am 1955; 37-A: 1243-1249.

3 Kane WJ: Scoliosis prevalence: a call for a statement of terms. Clin Orthop Relat Res 1977; 126: 43-46.

4 Bonaiti C, Feingold J, Briard ML, Lapeyre F, Rigault P, Guivarch J: (Genetics of idiopathic scoliosis). Helv Paediatr Acta 1976; 31: 229-240.

5 Kesling KL, Reinker KA: Scoliosis in twins. A meta-analysis of the literature and report of six cases. Spine (Phila Pa 1976) 1997; 22: 2009-2014; discussion 2015.

6 Riseborough EJ, Wynne-Davies R: A genetic survey of idiopathic scoliosis in Boston, Massachusetts. J Bone Joint Surg Am 1973; 55: 974-982.

7 Axenovich TI, Zaidman AM, Zorkoltseva IV, Tregubova IL, Borodin PM: Segregation analysis of idiopathic scoliosis: demonstration of a major gene effect. Am J Med Genet 1999; 86: 389-394.

8 Chan V, Fong GC, Luk KD et al: A genetic locus for adolescent idiopathic scoliosis linked to chromosome 19p13.3. Am J Hum Genet 2002; 71: 401-406.

9 Alden KJ, Marosy B, Nzegwu N, Justice CM, Wilson AF, Miller NH: Idiopathic scoliosis: identification of candidate regions on chromosome 19p13. Spine (Phila Pa 1976) 2006; 31: 1815-1819.

10 Salehi LB, Mangino M, De Serio S et al: Assignment of a locus for autosomal dominant idiopathic scoliosis (IS) to human chromosome 17p11. Hum Genet 2002; 111: 401-404.

11 Ocaka L, Zhao C, Reed JA et al: Assignment of two loci for autosomal dominant adolescent idiopathic scoliosis to chromosomes 9q31.2-q34.2 and 17q25.3-qtel. J Med Genet 2008; 45: 87-92.

12 Gurnett $C A$, Alaee F, Bowcock $A$ et al: Genetic linkage localizes an adolescent idiopathic scoliosis and pectus excavatum gene to chromosome $18 \mathrm{q}$. Spine (Phila Pa 1976) 2009; 34: E94-E100.

13 Kong A, Gudbjartsson DF, Sainz J et al: A high-resolution recombination map of the human genome. Nat Genet 2002; 31: 241-247.

14 Clerget-Darpoux F, Babron MC, Bonaiti-Pellie C: Power and robustness of the linkage homogeneity test in genetic analysis of common disorders. J Psychiatr Res 1987; 21: 625-630.

15 Abecasis GR, Cherny SS, Cookson WO, Cardon LR: Merlin-rapid analysis of dense genetic maps using sparse gene flow trees. Nat Genet 2002; 30: 97-101.

16 Clerget-Darpoux F, Babron MC, Prum B, Lathrop GM, Deschamps I, Hors J: A new method to test genetic models in HLA associated diseases: the MASC method. Ann Hum Genet 1988; 52: 247-258.

17 Strauch K, Fimmers R, Baur MP, Wienker TF: How to model a complex trait. 2. Analysis with two disease loci. Hum Hered 2003; 56: 200-211.

18 Kuklo TR, Potter BK, Polly Jr DW, O'Brien MF, Schroeder TM, Lenke LG: Reliability analysis for manual adolescent idiopathic scoliosis measurements. Spine (Phila Pa 1976) 2005; 30: 444-454.

19 Dang NR, Moreau MJ, Hill DL, Mahood JK, Raso J: Intra-observer reproducibility and interobserver reliability of the radiographic parameters in the Spinal Deformity Study Group's AIS Radiographic Measurement Manual. Spine (Phila Pa 1976) 2005; 30: 1064-1069.

20 Gao X, Gordon D, Zhang D et al: CHD7 gene polymorphisms are associated with susceptibility to idiopathic scoliosis. Am J Hum Genet 2007; 80: 957-965.

21 Sharma S, Gao X, Londono D et al: Genome-wide association studies of adolescent idiopathic scoliosis suggest candidate susceptibility genes. Hum Mol Genet 2011, e-pub ahead of print 7 January 2011. 